\title{
Relating the Freiheitssatz to the asymptotic behavior of a group
}

\author{
Francisco F. Lasheras and Ranja Roy
}

\begin{abstract}
We are concerned with the implications of the Freiheitssatz property for certain group presentations in terms of proper homotopy invariants of the underlying group, by describing its fundamental pro-group. A finitely presented group $G$ is said to be properly 3 -realizable if it is the fundamental group of a finite 2-dimensional CW-complex whose universal cover has the proper homotopy type of a 3-manifold. We show that if an infinite finitely presented group $G$ is given by some special kind of presentation satisfying the Freiheitssatz, then $G$ is semistable at infinity and properly 3 -realizable. In particular, this applies to groups given by a staggered presentation.
\end{abstract}

\section{Introduction}

For most of the paper, we will be working within the category of locally finite CW-complexes and proper maps between them, i.e., maps with the property that the inverse image of every compact subset is compact. Two such spaces are said to be proper homotopy equivalent if they are homotopy equivalent and all homotopies involved are proper.

Let $L$ be a locally finite $\mathrm{CW}$-complex. A proper map $\omega:[0, \infty) \longrightarrow L$ is called a proper ray in $L$. We say that two proper rays $\omega$ and $\omega^{\prime}$ define the same end $e$ if their restrictions to the natural numbers $\left.\omega\right|_{\mathbb{N}}$ and $\left.\omega^{\prime}\right|_{\mathbb{N}}$ are properly homotopic. The $\mathrm{CW}$-complex $L$ is said to be semistable at the end $e$ if any two proper rays $\omega$ and $\omega^{\prime}$ defining the same end $e$ are in fact properly homotopic. $L$ is semistable at infinity if it is semistable at each end.

Let $G$ be an infinite finitely presented group and $K$ be a finite 2-dimensional CW-complex with $\pi_{1}(K) \cong G$. Some of the proper homotopy invariants of the universal cover $\widetilde{K}$ of $K$ turn out to be invariants of the group $G$, for they do not depend on the choice of $K$. By the number of ends of $G$ we mean the number of

Mathematics Subject Classification (2010): Primary 57M07; Secondary 57M10, 57M20.

Keywords: Freiheitssatz, proper homotopy, ends of groups, semistable at infinity, fundamental pro-group, properly 3-realizable, 3-manifold. 
ends of $\widetilde{K}$ which equals 1,2 , or $\infty$ (finite groups have 0 ends, see [15] and [29]), and the group $G$ is said to be semistable at infinity if the universal cover $\widetilde{K}$ is so. On the other hand, $G$ is said to be properly 3 -realizable if the universal cover of the wedge $K \vee S^{2}$ is proper homotopy equivalent to a 3 -manifold. In some cases one may disregard the 2-sphere (see [1]). Notice that as $K$ is the Cayley complex associated to a finite presentation of $G$, then $\widetilde{K}$ is always proper homotopy equivalent to a 4-manifold, as $\widetilde{K}$ (as well as $\widehat{K \vee S^{2}}$ ) can be properly embedded in $\mathbb{R}^{4}$ (see also [4]), and hence it is natural to ask whether one can do better. See [9] and [20] for a source of examples of properly 3-realizable groups.

At the time of writing it is unknown whether all finitely presented groups are semistable at infinity. On the other hand, there are finitely presented groups which are not properly 3-realizable (see [5] and [13]).

Both properties above have implications in the theory of cohomology of groups. It is a long standing conjecture (attributed to Hopf) that $H^{2}(G ; \mathbb{Z} G)$ is free abelian for every finitely presented group $G$. Geoghegan and Mihalik [16] showed that if $G$ is semistable at infinity then $H^{2}(G ; \mathbb{Z} G)$ is free abelian. An isomorphism $H_{c}^{2}(\widetilde{K} ; \mathbb{Z}) \cong H^{2}(G ; \mathbb{Z} G) \oplus$ (free abelian $)$ is also shown in [16], for any finitely presented group $G$ and any finite 2 -dimensional CW-complex $K$ with $\pi_{1}(K) \cong G$. If $G$ is properly 3 -realizable, the cohomology group with compact support $H_{c}^{2}(\widetilde{K} ; \mathbb{Z})$ is free abelian by manifold duality arguments, and hence so is $H^{2}(G ; \mathbb{Z} G)$.

In order to state the main results, we first need some notation and some definitions. From now on, $X$ will be a finite set and $R$ will consist of a finite collection of cyclically reduced words in the free group $F(X)$.

Definition 1.1. We say that the presentation $\langle X ; R\rangle$ satisfies the Freiheitssatz (abbreviated as FHS) if any subset $Y \subset X$ not containing all the generators involved in any of the relators freely generates a free subgroup.

It is a well known result of Magnus [23] that one-relator group presentations have this property. There is an extensive work in the literature extending this result of Magnus to one-relator products. It is worth mentioning that for any finite presentation $\langle X ; R\rangle$ with $\operatorname{card}(X)>\operatorname{card}(R)$ there is some subset of generators of cardinality $\operatorname{card}(X)-\operatorname{card}(R)$ freely generating a free subgroup (see [28]).

Remark 1.2. Given a group presentation $\mathcal{P}=\langle X ; R\rangle$, we will denote by $K_{\mathcal{P}}$ the standard 2-dimensional CW-complex associated to this presentation (i.e., its Cayley complex). Namely, the 1 -skeleton $K_{\mathcal{P}}^{1}$ of $K_{\mathcal{P}}$ is a bouquet of circles consisting of a 1-cell $e_{x}$ for each generator $x \in X$, all of them sharing a single vertex in $K_{\mathcal{P}}$. Finally, $K_{\mathcal{P}}$ is obtained from $K_{\mathcal{P}}^{1}$ by attaching 2-cells $d_{r}$, one for each relator $r \in R$, via $(\mathrm{PL})$ maps $f_{r}: S^{1} \longrightarrow K_{\mathcal{P}}^{1}$ which spell out the words in $R$. Let $p: \widetilde{K_{\mathcal{P}}} \longrightarrow K_{\mathcal{P}}$ be the universal covering map. Given $Y \subset X$ and the subgraph $J \subset K_{\mathcal{P}}^{1}$ corresponding to those generators in $Y$, one can check that $Y$ freely generates a free subgroup if and only if $p^{-1}(J) \subset \widehat{K_{\mathcal{P}}}$ consists of a disjoint union of trees.

From now on, all CW-complexes will be PL CW-complexes, in the sense of $\S 1.4$ of [19]. Let us recall the notion of strong proper homotopy equivalence between 
2-dimensional (PL) CW-complexes introduced in [8]. Note that this notion is a (proper) generalization of the notion of simple homotopy equivalence within the category of 2-dimensional (PL) CW-complexes.

Definition 1.3. Let $L$ be a 2-dimensional (PL) CW-complex and let $d^{n} \subset e^{n+1}$ be cells of $L$. An elementary internal collapse $(e, d)$ in $L$ consists of "collapsing" the cell $e$ through its face $d$, even if $d$ is not a free face of $e$ within the entire complex $L$. Of course, this implies dragging all the cells adjacent to that face thus producing a new $\mathrm{CW}$-complex proper homotopy equivalent to $L$. We will refer to any of the inverses of an elementary internal collapse as an elementary internal expansion.

Definition 1.4. We define two (possibly infinite) 2-dimensional CW-complexes to be strongly proper homotopy equivalent if one is obtained from the other by a (possibly infinite) sequence of elementary internal collapses and/or expansions, in such a way that the resulting homotopy equivalence is a proper homotopy equivalence.

Definition 1.5. An infinite finitely presented group $G$ is said to have a fundamental pro-group of telescopic type at each end if there is a finite presentation $\mathcal{P}$ of $G$ and a $C W$-complex $L$ (proper homotopy equivalent to $\widetilde{K_{\mathcal{P}}}$ ) together with an exhaustion by compact subsets $C_{1} \subset C_{2} \subset \cdots \subset L$ such that, for any choice of base ray, the inverse sequence of groups

$$
0=\pi_{1}(L) \leftarrow \pi_{1}\left(L-C_{1}\right) \leftarrow \pi_{1}\left(L-C_{2}\right) \leftarrow \cdots
$$

is equivalent (i.e., pro-isomorphic) to a telescopic tower (see $\S 2$ ). In particular, $G$ is semistable at infinity.

Definition 1.6. If, in addition, the CW-complex $L$ above satisfies $\operatorname{dim}(L)=2$ and the following two conditions hold:

(i) the proper homotopy equivalence $\widetilde{K_{\mathcal{P}}} \longrightarrow L$ can be taken to be a strong proper homotopy equivalence;

(ii) the tower (1.1) is already a telescopic tower, and the subsets $C_{i} \subset L$ are all finite simply connected subcomplexes;

then the group $G$ is said to have a nice fundamental pro-group at each end. Furthermore, if the presentation $\mathcal{P}$ of $G$ satisfies the FHS, then we define $\mathcal{P}$ as a special presentation of $G$.

We will refer to a collection of subcomplexes $C_{1} \subset C_{2} \subset \cdots \subset L$ satisfying condition (ii) above as a nice filtration of the 2-dimensional simply connected CW-complex $L$.

Remark 1.7. Those groups which admit a one-relator group presentation $\mathcal{P}$ have a nice fundamental pro-group at each end (see [8]), and in this case $\mathcal{P}$ is special. Observe that the results in [8] make use of an additional CW-complex $L$, thus motivating Definitions 1.5 and 1.6. 
Remark 1.8. From the proper homotopy viewpoint, having a nice fundamental pro-group at each end can be regarded as a stronger property than the quasi simply filtered (QSF) property of Brick and Mihalik (see [3]) or the weakly geometrically simply connected (WGSC) property of Funar (see [12] and [14]). More precisely, $G$ is QSF if for any finite CW-complex $K$ with $\pi_{1}(K) \cong G$, the universal cover $\widetilde{K}$ admits an exhaustion which can be "approximated" by finite simply connected CW-complexes, i.e., for every finite subcomplex $A \subset \widetilde{K}$ there is a cellular map $f$ : $L \longrightarrow \widetilde{K}$ from a finite simply connected CW-complex $L$ which is a homeomorphism on $f^{-1}(A)$. On the other hand, $G$ is WGSC if for some $K$ as above, $\widetilde{K}$ has in fact an exhaustion by finite simply connected subcomplexes.

Remark 1.9. In [9] it was shown that for any two infinite finitely presented groups $G$ and $H$, the direct product $G \times H$ has a fundamental pro-group of telescopic type (at its single end). Moreover, one can easily derive from this that if $G$ and $H$ are both WGSC, then $G \times H$ has a nice fundamental pro-group at its end.

We generalize Theorem 1.2 in [20] in the 1-ended case to the following general result:

Theorem 1.10. Let $G$ be an infinite finitely presented group. If $G$ has a fundamental pro-group of telescopic type at each end, then $G$ is properly 3-realizable.

Remark 1.11. The converse of Theorem 1.10 has been proved recently in [13] under the QSF condition; in fact, it is conjectured there (as the "3-dimensional homotopy covering conjecture") that all properly 3-realizable groups are semistable at infinity and have a fundamental pro-group of telescopic type at each end. On the other hand, assuming semistability, the converse of Theorem 1.10 (at least in the 1-ended case) has been announced in [5], as an application of the work of Brin and Thickstun.

As a consequence of Theorem 1.10 (together with Remark 1.7), we answer Conjecture 1.5 in [8] in the affirmative; namely:

Corollary 1.12. All finitely generated one-relator groups are properly 3-realizable.

The following result provides another method of constructing new groups having a nice fundamental pro-group at each end, and it can be seen as a step forward in the study of the relationship between the FHS property and the asymptotic behavior of a group.

Theorem 1.13. Let $G$ and $H$ be finitely presented groups having nice fundamental pro-groups at each end, and assume $\mathcal{P}=\langle X ; R\rangle$ and $\mathcal{Q}=\langle Y ; S\rangle$ are special presentations of $G$ and $H$, respectively. Let $V \subset X$ and $W \subset Y$, together with a bijection $\eta: V \longrightarrow W$, be subsets not containing all the generators involved in any of the relators of the corresponding presentation. Then, the corresponding amalgamated product $G *_{F} H$ associated with $\eta$ (over a free group of rank $\operatorname{card}(V)=$ $\operatorname{card}(W))$ also has a nice fundamental pro-group at each end. Moreover, the obvious presentation for $G *_{F} H$ is also special. 
Since Theorem 1.13 can be applied repeatedly, it seems natural to consider the class $\mathcal{C}$ of all finitely generated one-relator groups together with those finitely presented groups which can be obtained by successive applications of Theorem 1.13 starting from any one-relator group presentations (see Remark 1.7), so that $\mathcal{C}$ is closed under amalgamated products of the type described in Theorem 1.13.

Remark 1.14. The first interesting examples of groups in the class $\mathcal{C}$ (other than one-relator groups) are those groups $G$ given by a presentation of the form $\langle X ; r, s\rangle$, where $r, s$ are cyclically reduced words so that $r \in F(Y), Y \subsetneq X$, and $s \in F(X)-F(Y)$ misses at least one generator in $Y$ which occurs in $r$. Indeed, one can obtain $G$ as an amalgamated product $\langle Y ; r\rangle *_{F}\langle(X-Y) \cup Z ; s\rangle$ over a free group of rank $\operatorname{card}(Z)$, where $Z \subsetneq Y$ is the subset consisting of those generators in $Y$ which occur in $s$.

Remark 1.15. From Remark 1.14, one can see that Higman's group $H$, with presentation $\left\langle a, b, c, d ; a^{-2} b^{-1} a b, b^{-2} c^{-1} b c, c^{-2} d^{-1} c d, d^{-2} a^{-1} d a\right\rangle$, is in $\mathcal{C}$. Indeed, $H$ can be expressed as an amalgamated product, as in Theorem 1.13, of two copies of $\left\langle x, y, z ; x^{-2} y^{-1} x y, y^{-2} z^{-1} y z\right\rangle$ over a free subgroup of rank 2 (see [18]).

On the other hand, we recall that $\langle X ; R\rangle$ is defined to be a staggered presentation if there is a subset $X_{0} \subset X$ so that both $R$ and $X_{0}$ are linearly ordered in such a way that: (i) each relator $r \in R$ contains some $x \in X_{0}$; (ii) if $r, r^{\prime}$ are relators with $r<r^{\prime}$, then $r$ contains some $x \in X_{0}$ that precedes all elements of $X_{0}$ occurring in $r^{\prime}$, and $r^{\prime}$ contains some $y \in X_{0}$ that comes after all those occurring in $r$ (see [22]).

Corollary 1.16. Every finitely presented group $G$ given by a staggered presentation $\mathcal{P}$ is in $\mathcal{C}$ and hence it has a nice fundamental pro-group at each end.

Proof. Suppose $\mathcal{P}=\langle X ; R\rangle$. We show that $G$ is in the class $\mathcal{C}$ by using induction on $\operatorname{card}(R)$. If $\operatorname{card}(R)=1$, this is true as $\mathcal{C}$ contains all one relator groups, by hypothesis. Next assume the result is true if $\operatorname{card}(R) \leq k$, and consider $G$ given by a staggered presentation $\mathcal{P}=\langle X ; R\rangle$ where $R$ has $k+1$ relators. Let $X_{0} \subset X$ satisfy the definition of a staggered presentation. Relabel the relators $r_{1}, r_{2}, \ldots, r_{k+1}$ in $R$ if necessary such that $r_{i}<r_{j}$ for $i<j$ with respect to the linear order in the set $R$. Then, there are generators $x_{i} \in X_{0}$ which occur in $r_{i}$, $i<k+1$, and precede all those occurring in $r_{k+1}$ with respect to the linear order in the set $X_{0}$. Let $Y \subset X$ be the subset containing all the generators that appear in $r_{1}, r_{2}, \ldots, r_{k}$. The relator $r_{k+1}$ has a generator $x_{k+1} \in X_{0}$ that comes after all those occurring in $r_{i}$ for all $i<k+1$, and hence $x_{k+1} \in X-Y$. Let $Z \subset Y$ be the subset of generators of $Y$ which also occur in $r_{k+1}$. Then, the group given by $\left\langle Y ; r_{1}, r_{2}, \ldots, r_{k}\right\rangle$ is a group in $\mathcal{C}$ by the induction hypothesis (as it has a staggered presentation) which has a free subgroup freely generated by $Z$, by Theorem 1.13 , as $x_{i} \notin Z$ for all $i<k+1$ (see also [22]). Finally, observe that $G$ is obtained as the amalgamated product $\left\langle Y ; r_{1}, r_{2}, \ldots, r_{k}\right\rangle *_{F}\left\langle(X-Y) \cup Z ; r_{k+1}\right\rangle$ over a free group $F$ of rank $\operatorname{card}(Z)$. Therefore, $G$ is in the class $\mathcal{C}$ and hence it has a nice fundamental pro-group at each end, by Theorem 1.13. 
Theorem 1.10 gives us the following:

Corollary 1.17. Every group $G \in \mathcal{C}$ is both semistable at infinity and properly 3-realizable.

Notice that the semistability at infinity for those groups in $\mathcal{C}$ already follows from the combination of [26] and [27], but we also give a description of (the proisomorphism type of) their fundamental pro-groups by means of Theorem 1.13.

Proposition 1.18. If $G \in \mathcal{C}$ is torsion-free then it has a finite aspherical presentation $\mathcal{P}$, i.e., the universal cover $\widetilde{K_{\mathcal{P}}}$ of $K_{\mathcal{P}}$ is contractible.

This extends a result of Dyer and Vasquez in [11] for torsion-free one-relator groups (see also Lyndon's Identity Theorem in [21] for a more algebraic version).

The key technical points of this paper are Lemma 3.2 and the construction of the CW-complex $\widehat{K_{\mathcal{R}}}$ in $\S 4$.

\section{Some preliminaries}

This section is intended to provide some of the background regarding the notion of fundamental pro-group and its connection with semistability at infinity. See [2], [15] and [25] for general reference and details.

In what follows, we will be working within the category tow-Gr whose objects are towers of groups, i.e., inverse sequences of groups and bonding homomorphisms

$$
\underline{A}=\left\{A_{n} ; f_{n}\right\}_{n \geq 0}=\left\{A_{0} \stackrel{f_{1}}{\longleftarrow} A_{1} \stackrel{f_{2}}{\longleftarrow} A_{2} \longleftarrow \cdots\right\} .
$$

A morphism in this category will be called a pro-morphism. More precisely, a pro-morphism from $\underline{A}=\left\{A_{n} ; f_{n}\right\}_{n \geq 0}$ to $\underline{B}=\left\{B_{n} ; g_{n}\right\}_{n \geq 0}$ is determined (up to a certain equivalence relation which allows us to think in terms of cofinal subsequences) by a function $\phi: \mathbb{Z}_{+} \longrightarrow \mathbb{Z}_{+}$and homomorphisms $h_{n}: A_{\phi(n)} \longrightarrow B_{n}$ $(n \geq 0)$ such that whenever $m \geq n$, there exists $k \geq \phi(n), \phi(m)$ making the following diagram commute:

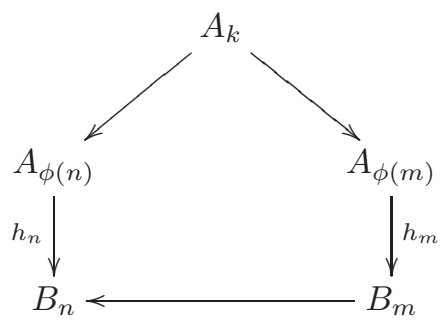

where the unmarked arrows are the obvious compositions of the corresponding bonding homomorphisms.

A tower $\underline{F}$ is a free tower if it is of the form

$$
\underline{F}=\left\{F_{0} \stackrel{i_{1}}{\longleftarrow} F_{1} \stackrel{i_{2}}{\longleftarrow} F_{2} \longleftarrow \cdots\right\}
$$


where $F_{i}=\left\langle B_{i}\right\rangle$ are free groups with bases $B_{i}$ such that $B_{i+1} \subset B_{i}$; the differences $B_{i}-B_{i+1}$ are finite and $\bigcap_{i=0}^{\infty} B_{i}=\emptyset$; and the bonding homomorphisms $i_{k}$ are given by the corresponding basis inclusions. On the other hand, a tower $\underline{P}$ is a telescopic tower if it is of the form

$$
\underline{P}=\left\{P_{0} \stackrel{p_{1}}{\longleftarrow} P_{1} \stackrel{p_{2}}{\longleftarrow} P_{2} \longleftarrow \cdots\right\},
$$

where $P_{i}=\left\langle D_{i}\right\rangle$ are free groups with bases $D_{i}$ such that $D_{i-1} \subset D_{i}$; the differences $D_{i}-D_{i-1}$ are finite (possibly empty); and the bonding homomorphisms $p_{k}$ are the obvious projections.

We will also use the full subcategory ( $\mathrm{Gr}$, tow-Gr) of Mor(tow-Gr) whose objects are arrows $\underline{A} \longrightarrow G$, where $\underline{A}$ is an object in tow-Gr and $G$ is a group regarded as a constant tower whose bonding maps are the identity. Morphisms in (Gr, tow-Gr) will also be called pro-morphisms.

Let $L$ be a connected locally compact topological space. Given a base ray $\omega$ in $L$ and a collection of compact subspaces $C_{1} \subset C_{2} \subset \cdots \subset L$ so that $L=\bigcup_{n=1}^{\infty} C_{n}$, the following tower, pro- $\pi_{1}(L, \omega)$,

$$
\left\{\pi_{1}(L, \omega(0)) \leftarrow \pi_{1}\left(L-C_{1}, \omega\left(t_{1}\right)\right) \leftarrow \pi_{1}\left(L-C_{2}, \omega\left(t_{2}\right)\right) \leftarrow \cdots\right\}
$$

can be regarded as an object in (Gr, tow-Gr) and it is called the fundamental progroup of $(L, \omega)$, where $\omega\left(\left[t_{i}, \infty\right)\right) \subset L-C_{i}$ and the bonding homomorphisms are induced by the inclusions. This tower does not depend (up to pro-isomorphism) on the sequence of subspaces $\left\{C_{i}\right\}_{i \geq 1}$. It is worth mentioning that if $\omega$ and $\omega^{\prime}$ are properly homotopic, then pro- $\pi_{1}(L, \omega)$ and pro- $\pi_{1}\left(L, \omega^{\prime}\right)$ are pro-isomorphic. On the other hand, a proper homotopy equivalence $f: L \longrightarrow L^{\prime}$ induces a proisomorphism between pro- $\pi_{1}(L, \omega)$ and pro- $\pi_{1}\left(L^{\prime}, f \circ \omega\right)$.

It is known that $L$ is semistable at the end determined by $\omega$ if and only if pro- $\pi_{1}(L, \omega)$ is pro-isomorphic to a tower where the bonding maps are surjections. Moreover, in this case $\pi_{1}^{e}(L, \omega)=\lim _{\longleftarrow} \operatorname{pro}-\pi_{1}(L, \omega)$ is a well defined and useful invariant which only depends (up to isomorphism) on the given end (see [17]). In a similar way, one can define objects in ( $\mathrm{Gr}$, tow-Gr) corresponding to the higher homotopy pro-groups of $(L, \omega)$.

Finally, given a finitely presented group $G$ and any finite 2-dimensional CWcomplex $K$ with $\pi_{1}(K) \cong G$, we will refer to the fundamental pro-group of $\widetilde{K}$ (at each end) as the fundamental pro-group of $G$ (at each end).

\section{Proof of Theorem 1.10}

Lemma 3.1 (Lemma 4.5 in [6]). Let $\psi: \underline{P} \rightarrow \underline{Q}$ be an epimorphism in (Gr, tow-Gr) (see $\S 2)$ where $\underline{P}$ is a telescopic tower and $\underline{Q} \overline{i s}$ a tower of free groups. Then, $\underline{Q}$ is also a telescopic tower.

The proof of Theorem 1.10 relies on the following result: 
Lemma 3.2. Let $G$ be a finitely presented group having a fundamental pro-group of telescopic type at each end, and assume $G$ splits as an amalgamated product $G_{0} *_{F} G_{1}$ (resp., an $H N N$-extension $H *_{F}$ ) over a finite group $F$. Then, each factor $G_{0}$ and $G_{1}$ (resp., the base group $H$ ) is either finite or else it also has a fundamental pro-group of telescopic type at each end.

Proof of Theorem 1.10. In the 1-ended case it is known that a group with such a fundamental pro-group is properly 3-realizable, by Theorem 1.2 in [20]. Note that the 2-ended groups are all known to be semistable at infinity and properly 3-realizable (see [1], [15]). Finite groups (i.e., 0-ended groups) are also known to be properly 3 -realizable.

Finally, in the infinitely many ended case, Stallings' theorem tells us that $G$ splits as an amalgamated product or an HNN-extension over a finite group (see [29] and [15]), and Dunwoody's accessibility result in [10] shows that the process of further factorization of the group in this way must terminate after a finite number of steps, after which each one of the factors can have at most one end. Moreover, by applying Lemma 3.2 repeatedly, one can see that each of these factors is either finite or else it also has a fundamental pro-group of telescopic type, whence they are all properly 3 -realizable by the above considerations. The conclusion now follows from the fact that amalgamated products (or HNN-extensions) over finite groups of properly 3-realizable groups are again properly 3-realizable (see [7]).

Proof of Lemma 3.2. Let $G_{0}, G_{1}$ be finitely presented groups and let $F$ be a finite group with presentation $\left\langle a_{1}, \ldots, a_{n} ; r_{1}, \ldots, r_{m}\right\rangle$. Consider monomorphisms $\varphi_{i}$ : $F \longrightarrow G_{i}(i=0,1)$, and denote by $G_{0} *_{F} G_{1}=\left\langle G_{0}, G_{1} ; \varphi_{0}\left(a_{i}\right)=\varphi_{1}\left(a_{i}\right), 1 \leq i \leq n\right\rangle$ the corresponding amalgamated product. Let $K_{0}$ and $K_{1}$ be finite 2-dimensional CW-complexes with $\pi_{1}\left(K_{i}\right) \cong G_{i}$, and let $f_{i}: \bigvee_{i=1}^{n} S^{1} \longrightarrow K_{i}(i=0,1)$ be cellular maps such that $\operatorname{Im} f_{i_{*}} \subseteq \pi_{1}\left(K_{i}\right)$ corresponds to the subgroup $\operatorname{Im} \varphi_{i} \subseteq G_{i}$. Let $L^{\prime}$ be the 2-dimensional $\mathrm{CW}$-complex associated to the given presentation of $F$, with 1-cells $e_{1}, \ldots, e_{n}$. Consider the adjunction spaces

$$
L=\left(\bigvee_{i=1}^{n} e_{i}\right) \times I \bigcup_{\left(\bigvee_{i=1}^{n} e_{i}\right) \times\left\{\frac{1}{2}\right\}} L^{\prime}
$$

(homotopy equivalent to $L^{\prime}$ ) and $M=L \cup_{f_{0} \times\{0\} \cup f_{1} \times\{1\}}\left(K_{0} \sqcup K_{1}\right)$. By Van Kampen's Theorem, $M$ is a finite 2-dimensional CW-complex with $\pi_{1}(M) \cong G_{0} *_{F}$ $G_{1}$. Let $\widetilde{M}$ be the universal cover of $M$ with covering map $p: \widetilde{M} \longrightarrow M$. Observe that $p^{-1}\left(K_{i}\right)$ consists of a disjoint union of copies of the universal cover $\widetilde{K}_{i}$ of $K_{i}$, since the inclusion $K_{i} \hookrightarrow M$ induces a monomorphism $G_{i} \hookrightarrow G_{0} *_{F} G_{1}$ between the fundamental groups, $i=0,1$ (see [22]). Also, $p^{-1}\left(L^{\prime}\right)$ consists of a disjoint union of copies of the universal cover $\widetilde{L}^{\prime}$ of $L^{\prime}$, as the inclusion $L^{\prime} \hookrightarrow M$ induces a monomorphism $F \hookrightarrow G_{0} *_{F} G_{1}$. Let $J$ be a connected component of $p^{-1}\left(\bigvee_{i=1}^{n} e_{i}\right) \subset$ $p^{-1}\left(L^{\prime}\right)$ and consider the finite 2-dimensional CW-complex $N^{\prime}=(J \times I) \cup_{J \times\left\{\frac{1}{2}\right\}} \widetilde{L}^{\prime}$. Thus, $\widetilde{M}$ comes together with the following data (see [29]):

(a) The disjoint unions $\bigcup_{p} \widetilde{K}_{0, p}$ and $\bigcup_{r} \widetilde{K}_{1, r}$ of copies of the vertex spaces $\widetilde{K}_{0}$ and $\widetilde{K}_{1}$ respectively. 
(b) The disjoint union $\bigcup_{p, q} N_{p, q}^{\prime}$ of copies of the edge space $N^{\prime}$.

(c) A one-to-one function $(p, q) \stackrel{\varphi}{\mapsto}(r, s)$ (given by the group action of $G_{0} *_{F} G_{1}$ on $\widetilde{M})$, so that for each pair of indices $(p, q), J \times\{0\} \subset N_{p, q}^{\prime}$ is glued to $\widetilde{K}_{0, p}$ via a lift $\widetilde{f}_{p, q}^{0}: J \times\{0\} \longrightarrow \widetilde{K}_{0, p}$ of the map $f_{0}$, and $J \times\{1\} \subset N_{p, q}^{\prime}$ is glued to $\widetilde{K}_{1, r}$ via a lift $\widetilde{f}_{r, s}^{1}: J \times\{1\} \longrightarrow \widetilde{K}_{1, r}$ of the map $f_{1}$.

Next, for each copy of $\widetilde{K}_{i}, i=0,1$, in $\widetilde{M}$ (written as $\widetilde{K}_{0, p}$ or $\widetilde{K}_{1, r}$ ), we take any one of the maps $\widetilde{f}_{\lambda, \mu}^{i}: J \times\{i\} \longrightarrow \widetilde{K}_{i}$. Observe that this map is null homotopic so that we can replace it (up to homotopy) by a constant map $g_{\lambda, \mu}^{i}: J \times\{i\} \longrightarrow \widetilde{K}_{i}$ whose image is a vertex inside $\operatorname{Im} \widetilde{f}_{\lambda, \mu}^{i}$. We do this replacement equivariantly using the group action of $G_{i}$ on $\widetilde{K}_{i}$. Since this action is properly discontinuous, the collection of all these homotopies gives rise to a proper homotopy equivalence between $\widetilde{M}$ and a new 2-dimensional CW-complex $\widehat{M}$, where $\widehat{M}$ is obtained from a collection of copies of $N=\Sigma J \cup_{J \times\left\{\frac{1}{2}\right\}} \widetilde{L}^{\prime}$ (" $\Sigma$ " stands for "suspension") and a collection of copies of $\widetilde{K}_{0}$ and $\widetilde{K}_{1}$ glued together appropriately through the suspension vertices of those copies of $\Sigma J \subset N$ (via the function $\varphi$ and the new maps $\left.g_{\lambda, \mu}^{i}, i=0,1\right)$. See the picture for a pictorial description of $\widehat{M}$ in the infinitely many ended case.

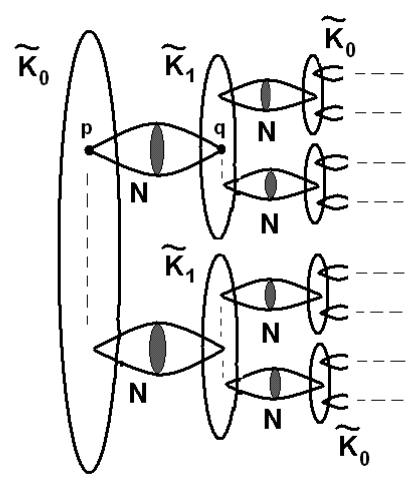

We choose a copy of $N$ in $\widehat{M}$ which we again call $N$. Let us denote by $\widetilde{K}_{0}$ and $\widetilde{K}_{1}$ those copies in $\widehat{M}$ of the universal covers of $K_{0}$ and $K_{1}$ which intersect $N$ at vertices $p \in \widetilde{K}_{0}$ and $q \in \widetilde{K}_{1}$, taken as base points. We consider filtrations by compact subsets $C_{i, 1} \subset C_{i, 2} \subset \cdots \subset \widetilde{K}_{i}$ with $p \in C_{0,1}$ and $q \in C_{1,1}$, and such that no vertex of $\widetilde{K}_{i}$ is in the boundary of any of the $C_{i, j}$ 's. These subsets $C_{i, j}$ 's may be taken as finite subcomplexes in some barycentric subdivision of $\widetilde{K}_{i}$. We proceed to build inductively a filtration by compact subsets $C_{1} \subset C_{2} \subset \cdots \subset \widehat{M}$. The subset $C_{1}$ consists of $N \cup C_{0,1} \cup C_{1,1}$. Assume $C_{n}$ is constructed. Then, $C_{n+1}$ is the union of: (i) $C_{n} \cup C_{0, n+1} \cup C_{1, n+1}$; (ii) the translates of $N$ which intersect $C_{n}$; and (iii) the translates of $C_{0, n+1}$ and $C_{1, n+1}$ on all those copies of the universal covers of $K_{0}$ and $K_{1}$ which intersect these translates of $N$ at a vertex (so that the corresponding base point on $\widetilde{K}_{i}$ is sent to the corresponding intersection vertex). 
Note that the fundamental pro-group of $G \cong G_{0} *_{F} G_{1}$ at each end is given by $\operatorname{pro}-\pi_{1}(\widetilde{M}) \cong \operatorname{pro}-\pi_{1}(\widehat{M}) \equiv\left\{\{1\} \longleftarrow \pi_{1}\left(\widehat{M}-C_{1}\right) \longleftarrow \pi_{1}\left(\widehat{M}-C_{2}\right) \longleftarrow \cdots\right\}$

where the base points are taken on any base ray determining the given end (see $\$ 2$ ). We will show that $\widetilde{K}_{0}$ (and hence $G_{0}$ ) has a fundamental pro-group of telescopic type at each end (the proof for $G_{1}$ is analogous). More precisely, for any given base ray in $\widetilde{K}_{0}$, we will define pro-morphisms:

$$
\operatorname{pro}-\pi_{1}\left(\widetilde{K}_{0}\right) \stackrel{\varphi}{\longrightarrow} \operatorname{pro}-\pi_{1}(\widehat{M}) \stackrel{\psi}{\longrightarrow} \operatorname{pro}-\pi_{1}\left(\widetilde{K}_{0}\right)
$$

so that $\psi \circ \varphi \equiv \mathrm{id}$. The result will then follow from Lemma 3.1, as $\varphi$ is a monomorphism, $\psi$ is an epimorphism and pro- $\pi_{1}(\widehat{M})$ is pro-isomorphic to a telescopic tower by hypothesis.

The pro-morphism $\varphi$ is determined by the inclusion-induced homomorphisms $\pi_{1}\left(\widetilde{K}_{0}-C_{0, j}\right) \longrightarrow \pi_{1}\left(\widehat{M}-C_{j}\right)$ (see $\left.\S 2\right)$. To define maps $h_{j}: \widehat{M}-C_{j} \longrightarrow \widetilde{K}_{0}-C_{0, j}$ we proceed as follows. Fix $p_{j} \in \widetilde{K}_{0}-C_{0, j}$. If $z \in \widehat{M}-C_{j}$ is in $\widetilde{K}_{0}-C_{0, j}$ then we set $h_{j}(z)=z$. Otherwise, define $h_{j}(z)$ in the following way. Let $\gamma$ be a path in $\widehat{M}$ from $z$ to $p$, and let $w$ be the vertex at which $\gamma$ meets $\widetilde{K}_{0}$ for the first time. If $w \notin C_{0, j}$ then we define $h_{j}(z)=w$; otherwise, we set $h_{j}(z)=p_{j}$. One can check that each $h_{j}$ is a continuous retraction, by the choice of the compact subsets $C_{0, j} \subset \widetilde{K}_{0}$. Observe that $h_{j}$ is not a proper map, as it maps noncompact subsets of each copy of the universal cover of $K_{i}$ (except for $\widetilde{K}_{0}$ ) to a point. However, as a loop in $\widehat{M}$ is a product (modulo change of base points) of loops each one of them living inside some copy of either $N$ or the universal cover of $K_{0}$ or $K_{1}$, one can check that these maps $h_{j}$ lead to commutative diagrams

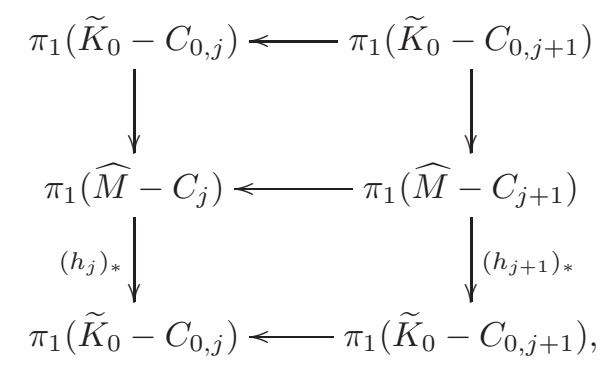

where the unmarked arrows are induced by the inclusions, and the composition of any two consecutive vertical arrows is the corresponding identity homomorphism. Finally, the pro-morphism $\psi:$ pro- $\pi_{1}(\widehat{M}) \longrightarrow$ pro- $\pi_{1}\left(\mathscr{K}_{0}\right)$ is that determined by the family of homomorphisms $\left\{\left(h_{j}\right)_{*}\right\}_{j \geq 1}$, and the conclusion follows.

In the case of an HNN-extension $H *_{F}=\left\langle H, t ; t^{-1} \psi_{0}\left(a_{i}\right) t=\psi_{1}\left(a_{i}\right), 1 \leq i \leq n\right\rangle$ (with monomorphisms $\psi_{i}: F \longrightarrow H, i=0,1$ ), let $K$ be a finite 2-dimensional CW-complex with $\pi_{1}(K) \cong H$ and $f_{i}: \bigvee_{i=1}^{n} S^{1} \longrightarrow K(i=0,1)$ cellular maps so that $\operatorname{Im} f_{i_{*}} \subseteq \pi_{1}(K)$ corresponds to the subgroup $\operatorname{Im} \psi_{i} \subseteq H$. Let $L$ be the 2-dimensional CW-complex constructed as above and consider the adjunction space $M=L \cup_{f_{0} \times\{0\} \cup f_{1} \times\{1\}} K$, with $\pi_{1}(M) \cong H *_{F}$. Then the proof is similar to the one given above for the amalgamated product. 


\section{Proof of Theorem 1.13 and Proposition 1.18}

Lemma 4.1 (Lemma 2.4 in [8]). Let $L$ be a 2-dimensional simply connected $\mathrm{CW}$ complex together with a nice filtration $C_{1} \subset C_{2} \subset \cdots \subset L$, and let $T_{i}, i \in I$, be a (locally finite) collection of trees inside L. There exists a new 2-dimensional $\mathrm{CW}$-complex $\widehat{L}$ together with a strong proper homotopy equivalence $L \longrightarrow \widehat{L}$, which leaves the $T_{i}$ 's unaltered, and a nice filtration $\widehat{C}_{1} \subset \widehat{C}_{2} \subset \cdots \subset \widehat{L}$ such that each $\widehat{C}_{n} \cap T_{i}(n \geq 1, i \in I)$ is either empty or a connected subtree.

Proof of Theorem 1.13. Let $\mathcal{P}=\langle X ; R\rangle$ and $\mathcal{Q}=\langle Y ; S\rangle$ be special presentations for the groups $G$ and $H$ respectively, and let $\eta: V \longrightarrow W$ be the given bijection between the subsets $V \subset X$ and $W \subset Y$ not containing all the generators involved in any of the relators of the corresponding presentation. Consider the (obvious) presentation $\mathcal{R}=\left\langle(X-V) \cup Y ; R^{\prime} \cup S\right\rangle$ for the corresponding amalgamated product $G *_{F} H$ associated with $\eta$, where $R^{\prime}$ is the collection of (cyclically reduced) words obtained from those in $R$ by replacing each $x \in V$ with $\eta(x) \in W$. The conclusion follows provided we can show that the 2-dimensional CW-complex $K_{\mathcal{R}}$ satisfies conditions (i) and (ii) in Definition 1.6. Moreover, we will show that the presentation $\mathcal{R}$ satisfies the FHS, whence $\mathcal{R}$ is a special presentation of $G *_{F} H$. The argument is divided into the following three steps.

1. The structure of the universal cover $\widetilde{K_{\mathcal{R}}}$. Observe that the CW-complex $K_{\mathcal{R}}$ is obtained from the $\mathrm{CW}$-complexes $K_{\mathcal{P}}$ and $K_{\mathcal{Q}}$ by identifying those 1-cells of $K_{\mathcal{P}}$ corresponding to the generators in $V$ with those 1-cells of $K_{\mathcal{Q}}$ corresponding to the generators in $W$, according to the bijection $\eta: V \longrightarrow W$. We will denote by $K_{0}$ and $K_{1}$ the resulting subcomplexes of $K_{\mathcal{R}}$ corresponding to $K_{\mathcal{P}}$ and $K_{\mathcal{Q}}$. Let $J=K_{0} \cap K_{1}$ and $p: \widetilde{K_{\mathcal{R}}} \longrightarrow K_{\mathcal{R}}$ be the universal covering map. Observe that $p^{-1}\left(K_{i}\right)$ consists of a disjoint union of copies of the universal cover of $K_{i}$ $(i=0,1)$, as the inclusions $K_{i} \hookrightarrow K_{\mathcal{R}}$ induce the monomorphisms $G \hookrightarrow G *_{F} H$ and $H \hookrightarrow G *_{F} H$ between the corresponding fundamental groups. Similarly, $p^{-1}(J)$ consists of a disjoint union of trees, each of them being a copy of the universal cover $T$ of $J$, as $J \hookrightarrow K_{\mathcal{R}}$ induces a monomorphism $F \hookrightarrow G *_{F} H$ as well. Thus, $\widetilde{K_{\mathcal{R}}}$ comes together with the following data:

(a) The disjoint unions $\bigcup_{p} \widetilde{K}_{0, p}$ and $\bigcup_{r} \widetilde{K}_{1, r}$ of copies of $\widetilde{K}_{0}$ and $\widetilde{K}_{1}$ respectively.

(b) The disjoint unions $\bigcup_{q} T_{0, p}^{q} \subset \widetilde{K}_{0, p}$ and $\bigcup_{s} T_{1, r}^{s} \subset \widetilde{K}_{1, r}$ of copies of the tree $T$, for each $p$ and $r$.

(c) A one-to-one function $(p, q) \stackrel{\varphi}{\mapsto}(r, s)$ (given by the group action of $G *_{F} H$ on $\left.\widetilde{K_{\mathcal{R}}}\right)$, so that for each pair of indices $(p, q)$, the tree $T_{0, p}^{q} \subset \widetilde{K}_{0, p}$ is identified with the tree $T_{1, r}^{s} \subset \widetilde{K}_{1, r}$.

It is easy to check from this description that the presentation $\mathcal{R}$ satisfies the FHS. For if $Z \subset(X-V) \cup Y$ is a subset not containing all the generators involved in any of the relators in $R^{\prime} \cup S, Z$ can be expressed as a union $Z=A^{\prime} \cup B$ where $A^{\prime} \subset(X-V) \cup W, B \subset Y$ and $A^{\prime} \cap B=Z \cap W$. Consider the subset $A=A^{\prime} \cap(X-V) \cup \eta^{-1}(Z \cap W) \subset X$. It is clear that the subset $A \subset X$ (respectively, $B \subset Y$ ) does not contain all the generators involved in any of the relators of 
the presentation $\mathcal{P}$ (respectively, the presentation $\mathcal{Q}$ ). Next, let $J_{0} \subset K_{0}\left(\equiv K_{\mathcal{P}}\right)$, $J_{1} \subset K_{1}\left(\equiv K_{\mathcal{Q}}\right)$ and $J_{Z} \subset K_{\mathcal{R}}$ denote the subgraphs corresponding to the subsets $A^{\prime}, B$ and $Z$ respectively, and let $q_{i}=p: \bigcup_{j} \widetilde{K}_{i, j} \subset \widetilde{K_{\mathcal{R}}} \longrightarrow K_{i} \subset K_{\mathcal{R}}(i=0,1)$ be the corresponding restriction maps, which are defined as the corresponding universal covering map $\widetilde{K}_{i, j} \longrightarrow K_{i}$ on each $\widetilde{K}_{i, j}$. Then, $p^{-1}\left(J_{Z}\right)$ consists of $q_{0}^{-1}\left(J_{0}\right)$ and $q_{1}^{-1}\left(J_{1}\right)$ glued together by identifying each connected subtree $q_{0}^{-1}\left(J_{0}\right) \cap T_{0, p}^{q} \subset \widetilde{K}_{0, p}$ with the corresponding connected subtree $q_{1}^{-1}\left(J_{1}\right) \cap T_{1, r}^{s} \subset \widetilde{K}_{1, r}$. Therefore, since $\mathcal{P}$ and $\mathcal{Q}$ both satisfy the FHS, each component of $q_{i}^{-1}\left(J_{i}\right)(i=0,1)$ is a tree, by Remark 1.2, and hence so is each component of $p^{-1}\left(J_{Z}\right)$. The conclusion follows again from Remark 1.2.

Remark 4.2. One can readily check from the description above that if $\widetilde{K_{\mathcal{P}}}$ and $\widetilde{K_{\mathcal{Q}}}$ are both contractible, then so is $\widetilde{K_{\mathcal{R}}}$.

2. Altering $\widetilde{K_{\mathcal{R}}}$ within its (strong) proper homotopy type. Since $\mathcal{P}$ and $\mathcal{Q}$ are special presentations of $G$ and $H$, for each copy $\widetilde{K}_{i, j}$ of $\widetilde{K}_{i}$ there is a 2 dimensional CW-complex $\widehat{K}_{i, j}$ together with a strong proper homotopy equivalence $h_{i, j}: \widetilde{K}_{i, j} \rightarrow \widehat{K}_{i, j}$ and a nice filtration $C_{i, 1}^{j} \subset C_{i, 2}^{j} \subset \cdots \subset \widehat{K}_{i, j}$ (see Definition 1.6).

Let $M_{i, j}^{k}$ denote the mapping cylinder of the map $T_{i, j}^{k} \longrightarrow h_{i, j}\left(T_{i, j}^{k}\right)$, and let $\pi_{i, j}^{k}: T_{i, j}^{k} \times I \longrightarrow M_{i, j}^{k}$ denote the map determined by the corresponding quotient map, with $\pi_{i, j}^{k}\left(T_{i, j}^{k} \times\{1\}\right)=h_{i, j}\left(T_{i, j}^{k}\right)$. Let $L_{i, j}=\widehat{K}_{i, j} \bigcup_{\left\{h_{i, j}\left(T_{i, j}^{k}\right)\right\}} \bigcup_{k} M_{i, j}^{k}$, and consider the subcomplexes

$$
D_{i, n}^{j}=C_{i, n}^{j} \cup \bigcup_{k} \pi_{i, j}^{k}\left(h_{i, j}^{-1}\left(C_{i, n}^{j} \cap h_{i, j}\left(T_{i, j}^{k}\right)\right) \times I\right) \subset L_{i, j}, \quad n \geq 1 .
$$

It is easy to check that $D_{i, 1}^{j} \subset D_{i, 2}^{j} \subset \cdots \subset L_{i, j}$ is a nice filtration of $L_{i, j}$, as each complement $L_{i, j}-D_{i, n}^{j}$ deformation retracts onto $\widehat{K}_{i, j}-C_{i, n}^{j}$ (using the mapping cylinder structure of each $M_{i, j}^{k}$ ), and the $C_{i, n}^{j}$ 's form a nice filtration of $\widehat{K}_{i, j}$. By Lemma 4.1, we can further assume that $D_{i, 1}^{j} \subset D_{i, 2}^{j} \subset \cdots \subset L_{i, j}$ already satisfies that each intersection $D_{i, n}^{j} \cap T_{i, j}^{k}(j, k, n \geq 1, i=0,1)$ is either empty or a connected subtree.

Finally, we denote by $\widehat{K_{\mathcal{R}}}$ the 2-dimensional CW-complex obtained as a quotient space from the disjoint union $\bigcup_{i, j} L_{i, j}$ by identifying each tree $T_{0, p}^{q} \subset L_{0, p}$ with the corresponding tree $T_{1, r}^{s} \subset L_{1, r}$, as in the construction of $\widetilde{K_{\mathcal{R}}}$. It is not hard to see that there is a strong proper homotopy equivalence $\widetilde{K_{\mathcal{R}}} \longrightarrow \widehat{K_{\mathcal{R}}}$, as follows. First, we expand each copy $T_{0, p}^{q} \equiv T_{1, r}^{s}$ (within $\widetilde{K_{\mathcal{R}}}$ ) of the tree $T$ to a product $T \times[-1,1]$, keeping the copy $\widetilde{K}_{0, p}$ of $\widetilde{K}_{0}$ attached to $T \times\{-1\}$ and the corresponding copy $\widetilde{K}_{1, r}$ of $\widetilde{K}_{1}$ attached to $T \times\{1\}$. Next, we perform all the elementary internal collapses and/or expansions needed for passing from each $T \times[-1,0] \cup \widetilde{K}_{0, p}$ to a copy of $L_{0, p}$ and from each $T \times[0,1] \cup \widetilde{K}_{1, r}$ to a copy of $L_{1, r}$ (starting with those needed for passing from each $\widetilde{K}_{i, j}$ to $\widehat{K}_{i, j}$ ). Thus, condition (i) in Definition 1.6 is satisfied with respect to $\mathcal{R}$ and $L=\widehat{K_{\mathcal{R}}}$. 
3. Building the required nice filtration for $\widehat{K_{\mathcal{R}}}$. Fix $i_{0} \in\{0,1\}$ and $j_{0} \geq 1$, and consider $D_{i_{0}, 1}^{j_{0}} \subset L_{i_{0} . j_{0}}$. Let $L_{i, j(1)}, \ldots, L_{i, j\left(p_{1}\right)}$ be those subcomplexes of $\widehat{K_{\mathcal{R}}}$ which intersect $L_{i_{0}, j_{0}}$ at points of $D_{i_{0}, 1}^{j_{0}}$. Take $N_{1} \geq 1$ such that $D_{i, N_{1}}^{j(m)} \cap L_{i_{0}, j_{0}} \supset$ $D_{i_{0}, 1}^{j_{0}} \cap L_{i, j(m)}$ (both intersections being connected subtrees by hypothesis), $1 \leq$ $m \leq p_{1}$. Set

$$
D_{1}=D_{i_{0}, 1}^{j_{0}} \cup\left(\bigcup_{m=1}^{p_{1}} D_{i, N_{1}}^{j(m)}\right) \subset \widehat{K_{\mathcal{R}}} .
$$

This $D_{1}$ is simply connected, as the various finite simply connected subcomplexes $D_{\alpha, \gamma}^{\beta}$ involved in $D_{1}$ intersect each other along connected subtrees of those trees used in the construction of $\widehat{K_{\mathcal{R}}}$ (see Step 2). Moreover, by the generalized Van Kampen's theorem (see [15], [29]), as the subcomplexes $L_{i, j} \subset \widehat{K_{\mathcal{R}}}$ are simply connected and knowing that each $D_{\alpha, \gamma}^{\beta}$ is a member of a nice filtration of the corresponding $L_{\alpha, \beta}$, one can check (choosing base points on any given base ray in $\widehat{K_{\mathcal{R}}}$ ) that $\pi_{1}\left(\widehat{K_{\mathcal{R}}}-D_{1}\right)$ is a free product of $\pi_{1}\left(L_{i_{0}, j_{0}}-D_{i_{0}, 1}^{j_{0}}\right)$ and $\pi_{1}\left(L_{i, j(m)}-D_{i, N_{1}}^{j(m)}\right)$, $1 \leq m \leq p_{1}$, together with an extra free group (of finite rank) coming from the intersection of $D_{i_{0}, 1}^{j_{0}}$ and each $D_{i, N_{1}}^{j(m)}$ with any of the other subcomplexes $L_{\alpha, \beta}$.

Consider now $D_{i_{0}, 2}^{j_{0}} \subset L_{i_{0}, j_{0}}$, and let $L_{i, j(1)}, \ldots, L_{i, j\left(p_{2}\right)}\left(p_{2} \geq p_{1}\right)$ be those subcomplexes of $\widehat{K_{\mathcal{R}}}$ which intersect $L_{i_{0}, j_{0}}$ at points of $D_{i_{0}, 2}^{j_{0}}$. Take $N_{2} \geq N_{1}$ such that $D_{i, N_{2}}^{j(m)} \cap L_{i_{0}, j_{0}} \supset D_{i_{0}, 2}^{j_{0}} \cap L_{i, j(m)}$ (both being connected subtrees), for $1 \leq m \leq p_{2}$. Next, for each $1 \leq m \leq p_{2}$, let $L_{i_{0}, j(m, 1)}, \ldots, L_{i_{0}, j\left(m, q_{m}\right)}$ be those subcomplexes of $\widehat{K_{\mathcal{R}}}$ which intersect $L_{i, j(m)}$ at points of $D_{i, N_{2}}^{j(m)}$. Take $N_{3} \geq N_{2}$ such that $D_{i_{0}, N_{3}}^{j(m, l)} \cap L_{i, j(m)} \supset D_{i, N_{2}}^{j(m)} \cap L_{i_{0}, j(m, l)}$ (both being connected subtrees), for $1 \leq l \leq q_{m}$. Set

$$
D_{2}=D_{i_{0}, 2}^{j_{0}} \cup\left(\bigcup_{m=1}^{p_{1}} D_{i, N_{2}}^{j(m)}\right) \cup\left(\bigcup_{m=1}^{p_{2}} \bigcup_{l=1}^{q_{m}} D_{i_{0}, N_{3}}^{j(m, l)}\right) \subset \widehat{K_{\mathcal{R}}}
$$

As before, one can check that $D_{2}$ is simply connected and $\pi_{1}\left(\widehat{K_{\mathcal{R}}}-D_{2}\right)$ is a finitely generated free group. Finally, repeating this process (starting with the successive $D_{i_{0}, n}^{j_{0}} \subset L_{i_{0}, j_{0}}$ and going each time one step further inside $\widehat{K_{\mathcal{R}}}$ ) we get a filtration $D_{1} \subset D_{2} \subset \cdots \subset \widehat{K_{\mathcal{R}}}$ by finite simply connected subcomplexes. Moreover, choosing base points on any given base ray, one can easily check that $\operatorname{rank}\left(\pi_{1}\left(\widehat{K_{\mathcal{R}}}-D_{n+1}\right)\right) \geq \operatorname{rank}\left(\pi_{1}\left(\widehat{K_{\mathcal{R}}}-D_{n}\right)\right)$ and the inclusion-induced homomorphism $\pi_{1}\left(\widehat{K_{\mathcal{R}}}-D_{n+1}\right) \longrightarrow \pi_{1}\left(\widehat{K_{\mathcal{R}}}-D_{n}\right)$ can be taken to be a projection between finitely generated free groups. Therefore, condition (ii) in Definition 1.6 is also satisfied and hence the conclusion follows.

Proof of Proposition 1.18. Let $\mathcal{C}^{\prime} \subset \mathcal{C}$ be the class of those groups which can be obtained by successive applications of Theorem 1.13 starting from any torsion-free one-relator group presentation. By Theorem 2.7 in $\S \mathrm{IV}$ of $[22], \mathcal{C}^{\prime}$ is precisely the subclass of all those groups in $\mathcal{C}$ which are torsion-free. The conclusion follows 
from the fact that, for any torsion-free one-relator group presentation $\mathcal{P}$, the universal cover $\widetilde{K_{\mathcal{P}}}$ of $K_{\mathcal{P}}$ is contractible (see [11] and [21]) together with successive applications of Remark 4.2.

\section{References}

[1] Ayala, R., Cárdenas, M., Lasheras, F. F. And Quintero, A.: Properly 3-realizable groups. Proc. Amer. Math. Soc. 133 (2004), no. 5, 1527-1535.

[2] Baues, H.-J. And Quintero, A.: Infinite homotopy theory. K-monographs in Mathematics 6, Kluver Academic Publishers, Dordrecht, 2001.

[3] Brick, S. G. And Minalik, M.: The QSF property for groups and spaces. Math. Z. 220 (1995), 207-217.

[4] Cárdenas, M., Fernández, T., Lasheras, F. F. And Quintero, A.: Embedding proper homotopy types. Colloq. Math. 95 (2003), no. 1, 1-20.

[5] Cárdenas, M., Lasheras, F. F. And Quintero, A.: On properly 3-realizable groups which are semistable. Preprint

[6] Cárdenas, M., Lasheras, F. F., Muro, F. and Quintero, A.: Proper L-S category, fundamental pro-groups and 2-dimensional proper co- $H$-spaces. Topology Appl. 153 (2005), 580-604.

[7] Cárdenas, M., Lasheras, F.F., Quintero, A. and Repovš, D.: Amalgamated products and properly 3-realizable groups. J. Pure Appl. Algebra 208 (2007), no. 1, 293-296.

[8] Cárdenas, M., Lasheras, F.F., Quintero, A. and Repovš, D.: Onerelator groups and proper 3-realizability. Rev. Mat. Iberoamericana 25 (2009), no. 2, 739-756.

[9] Cárdenas, M., Lasheras, F.F: And Roy, R.: Direct products and properly 3-realisable groups. Bull. Austral. Math. Soc. 70 (2004), 199-205.

[10] Dunwoody, M. J.: The accessibility of finitely presented groups. Invent. Math. $\mathbf{8 1}$ (1985), 449-457.

[11] Dyer, E. And Vasquez, A. T.: Some small aspherical spaces. J. Austral. Math. Soc. 16 (1973), 332-352.

[12] Funar, L. ANd Gadgil, S.: On the geometric simple connectivity of open manifolds. Int. Math. Res. Not. 2004, no. 24, 1193-1248.

[13] Funar, L., Lasheras, F. F. And Repovš, D.: Groups which are not properly 3-realizable. Rev. Mat. Iberoamericana 28 (2012), no. 2, 401-414.

[14] Funar, L. And Otera, D. E.: On the wgsc and qsf tameness conditions for finitely presented groups. Groups Geom. Dyn. 4 (2010), 549-596.

[15] Geoghegan, R.: Topological methods in group theory. Graduate Texts in Mathematics 243, Springer, New York, 2008.

[16] Geoghegan, R. And Mihalik, M.: Free abelian cohomology of groups and ends of universal covers. J. Pure Appl. Algebra 36 (1985), 123-137.

[17] Geoghegan, R. and Minalik, M.: The fundamental group at infinity. Topology 35 (1996), no. 3, 655-669.

[18] Higman, G.: A finitely generated infinite simple group. J. London Math. Soc. 26 (1951), 61-64. 
[19] Hog-Angeloni, C., Metzler, W. and Sieradski, A. J.: Two-dimensional homotopy and combinatorial group theory. London Mathematical Society Lecture Notes Series 197, Cambridge University Press, Cambridge, 1993.

[20] Lasheras, F. F.: Ascending HNN-extensions and properly 3-realisable groups. Bull. Austral. Math. Soc. 72 (2005), 187-196.

[21] Lyndon, R. C.: Cohomology theory of groups with a single defining relation. Ann. of Math. (2) $\mathbf{5 2}$ (1950), 650-665.

[22] Lyndon, R. C. And Schupp, P. E.: Combinatorial group theory. Ergebnisse der Mathematik und ihrer Grenzgebiete 89, Springer-Verlag, Berlin-New York, 1977.

[23] Magnus, W.: Über diskontinuierliche Gruppen mit einer definierenden Relation. (Der FHS). J. Reine Angew. Math. 163 (1930), 141-165.

[24] Magnus, W., Karras, A. And Solitar, D.: Combinatorial group theory: Presentations of groups in terms of generators and relations. Interscience Publishers [John Wiley \& Sons], New York-London-Sydney, 1966.

[25] Mardešić, S. And Segal, J.: Shape theory. The inverse system approach. NorthHolland Mathematical Library 26, North-Holland, Amsterdam-New York, 1982.

[26] Mihalik, M. And Tschantz, S.: One relator groups are semistable at infinity. Topology 31 (2000), no. 4, 801-804.

[27] Minalik, M. And Tschantz, S.: Semistability of amalgamated products and HNNextensions. Mem. Amer. Math. Soc. 98 (1992), no. 471.

[28] Romanovskit, N. S.: Free subgroups of finitely presented groups. Algebra and Logic 16 (1977), 62-68.

[29] Scott, P. And Wall, C. T. C.: Topological methods in group theory. In Homological group theory (Proc. Sympos., Durham, 1977), 137-203. London Math. Soc. Lecture Notes Ser. 36, Cambridge Univ. Press, Cambridge-New York, 1979.

Received January 4, 2011.

Francisco F. Lasheras: Departamento de Geometría y Topología, Universidad de Sevilla, Apdo 1160, 41080-Sevilla, Spain.

E-mail: lasheras@us.es

RANJA RoY: New York Institute of Technology, Old Westbury, NY 11568-8000, USA. E-mail: rroy@nyit.edu

This work was partially supported by the project MTM2010-20445. The authors acknowledge remarks and suggestions by Antonio Quintero. 